\title{
THE TRANSFORMATION FROM FK4 TO FK5 AND THE DEFINITION OF UT
}

\author{
B.X.XU, Y.F.XIA, T.Y.HUANG, C.H.HAN \\ Department of Astronomy \\ Nanjing University \\ Nanjing, 210008 \\ People's Republic of China
}

\begin{abstract}
The different procedures of the transforation from FK4 to FK5 are reviewed. With these procedures some numerical examples for selected FK4 stars are given. Basing on the original work of Fricke (1985), we prefer to select the procedure developed by Standish (1982). One of the main reasons for the procedure of Aoki et al. (1983) is to consist with the new definition of UT. Since the corrections "FK5 - FK4" for many stars are significant, even if adopting this procedure, it is still impossible to avoid the effect of the right ascension discontinuities on UT.
\end{abstract}

\section{Introduction}

According to the resolution adopted by IAU in 1976, the FK4 should replaced by the FK5 from 1984 January 1 onward. The transformation from FK4 to FK5 includes the following processes: (1) eliminate the E terms of aberration. (2) correct the equinox error and its motion. (3) correct the proper motion for the new precession constant. (4) change the unit of the time from tropical to Julian centuries. (5) transform the positions and proper motions to J2000.0. (6) apply the systematic and individual corrections ("FK5 - FK4"). Because the "FK5 - FK4" were not available for the year 1984-1987, there was a transitional phase in which only the first five procedures have been applied.

For the transitional transformation several approaches have been developed by Standish (1982), Aoki et al. (1983), Lederle and Schwan (1984), Smith et al. (1989), Yallop et al. (1989), Murray (1989) and Soma et al. (1989). Among these there are two main differences: (1) The equinox correction is applied in the fixed frame (Standish) or in the rotating frame (Aoki et al.). (2) The transfer from FK4 to FK5 is performed at B1950.0 (Standish) or at 1984 January 1 (Aoki et al.).

In Sect.2 we suggest a criterion to review the differences. In Sect.3 some numerical examples are given for comparison. In Sect.4 we discuss the problem whether the transformation should or could consist with new definition of UT. 


\section{The Review of Different Procedures}

In the transformation the equinox error of FK4 and the correction of precession constant, which are determined by Fricke, are adopted; therefore the procedure of the transformation should base on the original work of Fricke (1985).

\subsection{THE DETERMINATION OF THE EQUINOX ERROR OF FK4}

Fricke adopted the following formula for determining the equinox error of FK4

$$
\Delta \alpha=E+f(\Delta \lambda, \Delta \varepsilon, \Delta h, \Delta k)
$$

where $\Delta \alpha=\left(\alpha_{0}\right)_{0}-\left(\alpha_{(0)}\right)_{c} .\left(\alpha_{(0)}\right)_{0}$ is the observed right ascension of the sun, and $\left(\alpha_{(0)}\right)_{c}$ is the computed value provided by an ephemeris. $\Delta \lambda, \Delta \varepsilon, \Delta h$ and $\Delta k$ are corrections to the "solar orbit".

Combining a series of observational results from the sun and members of the planetary system, Fricke obtained the equinox error of FK4. Soma et al. (1989) show that the equinox correction $E$ of Fricke is determined in the frame of date. On the contrary, we think that the equinox correction $E$ so determined is in the fixed frame. In fact, deriving a numerical value of $E$ needs the observational results of one year or even several years. They all must be reduced to the FK4 system. Moreover, the solar ephemeris is also founded in the fixed frame. It is convincing that Fricke oneself gave the transformation from FK 4 to FK5.

\subsection{THE DETERMINATION OF PRECESSION CORRECTION}

Fricke made use of proper motion, $\mu_{F K^{4}}$, of $\mathrm{FK} 4$ stars to determine the precession correction. If the effect of precessional error and zero point error on proper motion is only considered, then the formula becomes

$$
\begin{aligned}
& \Delta\left(\mu_{\alpha} \cos \delta\right)=\Delta k \cdot \cos \delta+\Delta n \cdot \sin \alpha \sin \delta \\
& \Delta \mu_{\delta}=\Delta n \cdot \cos \alpha
\end{aligned}
$$

where $\Delta l=\Delta p_{1} \cdot \cos \varepsilon-\Delta \lambda-\Delta e, \Delta n=\Delta p_{1} \cdot \sin \varepsilon . \quad p_{1}$ and $\lambda$ are the lunisolar and planetary precessions respectively. $\Delta e$ is "equinox motion" of $\mathrm{FK} 4$, and it corresponds to the derivative of $E$ in $E q .(1)$.

From the above consideration it appears that Fricke took no account of the effect of equinox correction on $\mu_{F K 4}$. Accordingly the effect of equinox correction should not be involved when the proper motion relative to new precession constant is corrected.

As is given in the abnve discussion, it is obvious that the procedure adopted by Standish is in keeping with the original work of Fricke. We insist that $\mathrm{E}_{\mathrm{q}}(3)$ in the paper of Soma. et al. be adopted.

\section{Numerical Examples and Comparison}

In order to compare numerical values obtained from different procedures of the transformation, we select four FK4 stars in the computation (from Table 1 of Smith et al.) 
All authors have no objection to the removal of the elliptic terms in aberration. Using the formula given by Lederle and Schwan (1984), we make first the correction to the catalog position. Applying steps (2), (3), (4) and (5) in the Introduction, we compute the transformation matrices by Standish (St), Aoki et al. (Ao) and Soma et al. (So) (see Soma et al. (1989), Eqs.(1),(2),(10)). Numerical examples for J2000.0 are given in Table 1 for selected four FK 4 stars.

Table 1. Numerical examples for J2000.0

\begin{tabular}{|c|c|c|c|c|c|}
\hline No. & Proc. & $\alpha$ & $\delta$ & P.M.R.A. & P.M.DEC! \\
\hline \multirow{3}{*}{10} & $\overline{\mathrm{St}}$ & $00^{h} 20^{m} 04^{s} 3088$ & $-64^{\circ} 52^{\prime} 29^{\prime \prime} 333$ & $26^{s} 8627$ & 116.284 \\
\hline & Ao & .3096 & .331 & .8647 & .286 \\
\hline & So & .3092 & .332 & .8635 & .286 \\
\hline \multirow{3}{*}{907} & $\overline{\mathrm{St}}$ & $02^{h} 31^{m} 49^{s} 8303$ & $+89^{\circ} 15^{\prime} 50^{\prime \prime} 655$ & $21^{8} 7784$ & -1.581 \\
\hline & Ao & .8113 & .659 & .7287 & .571 \\
\hline & So & .8227 & .658 & .7634 & .576 \\
\hline \multirow{3}{*}{923} & $\mathrm{St}$ & $21^{h} 08^{m} 46^{s} 0510$ & $-88^{\circ} 57^{\prime} 23^{\prime \prime} 666$ & $8 \cdot^{\circ} 4080$ & 0.180 \\
\hline & Ao & .0673 & .669 & .4481 & .172 \\
\hline & So & .0601 & .667 & .4263 & .177 \\
\hline \multirow{3}{*}{1307} & $\overline{\mathrm{St}}$ & $11^{h} 52^{m} 58 .^{s} 7456$ & $+37^{\circ} 43^{\prime} 07^{\prime \prime}, 460$ & $33 \cdot^{3} 7149$ & $-581^{\prime \prime} 214$ \\
\hline & Ao & .7460 & .459 & .7157 & .215 \\
\hline & So & .7458 & .459 & .7152 & .216 \\
\hline
\end{tabular}

We note from Table 1 that

(1) For non-circumpolar stars the differences of J2000.0 positions and proper motions obtained by four approaches can be omitted.

(2) För circumpolar stars the four approaches differ systematically by as much as 0.02 and 0:05 per century in $\mathrm{J} 2000.0$ right ascensions and proper motions in right ascension respectively.

Another transformation on the removal of the E terms of aberration from the J2000.0 mean place was adopted in Merit Standard (1983) and Astronomical Alamanac (1984). We examine the effect of epoch change of stellar reference system on $\mathrm{E}$ terms of aberration. We find that for circumpolar stars the effect of 50-year epoch change of the reference system on $E$ terms of aberration may reach to $0^{s} 4$ and 0.07 respectively. The effect is far larger than the differences from different procedures of the transformation in Table 1.

\section{The Review of the New Definition of UT}

Since the Eighteenth General Assembly of the IAU in 1982, there have been many reviews on the new definition of UT. Its main defects are as follows: (1) Lack of a clear physical cocept of UT. (2) Involving some determined constants. (3) Not applicable to the new techniques.

Aoki et al. (1983) emplasized that the transformation from FK4 to FK5 should consist with the new definition of UT, and demanded that the positions and proper motions of all FK4 stars referred to the new and old systems respectively are the same at 1984 January 
1. This is logically unreasonable. In fact, the fundamental plane and origin of a stellar reference system can be defined, whereas the positions and proper motions of all stars cannot be specified. Their numerical values can only be determined by observations.

As is given in the Introduction, the transformation from FK4 to FK5 involves 6 steps. The systematic and individual correctons of $\mathrm{FK} 4$ stars should be applied to the result of transitional transformation. According to "Corrections FK5 - FK4" published by Wielen et al. (1987), for $1984 \Delta \alpha$ of 69 stars are larger than 0.1 , among them $\Delta \alpha$ of 23 stars are larger than 0.2 . It is obvious that the effects of the systematic and individual corrections for many stars on the determination of UT cannot be omitted at the beginning of 1984 .

From 1988 onwards the IERS system no longer includes the optical observations. So it seems that the new definition of UT is unnecessary in IERS Standard. We suggest that the Working Group of the Reference System should rediscuss the definition of UT.

\section{References}

Aoki, S., Soma, M., Kinoshita, H., Inoue, K.,: 1983, Astron. Astrophys., 128, 263.

Astronomical Alamanac (1984), U.S. GPO, Washington, DC.

Fricke, W.,:198.5, Celest. Mech., 36, 207.

Fricke, W., Schwan, H., Lederle, T.,: 1988, Fifth Fundamental Catalogue (FK5), Veröff.

Astron. Recheninst., Heidelberg, No. 32.

Lederle, T., Schwan, H.,: 1984, Astron. Astrophys., 134, 1.

McCarthy, D. D., (ed.): 1989, IERS Standards, Observatoire de Paris.

Melbourne, W., (ed.): 1983, Project MERIT Standards, U.S. Naval Observatory Circular No.167.

Murray, C. A.,: 1989, Astron. Astrophys., 218, 325.

Smith, C. A., Kaplan, G. H., Hughes, J. A., Seidelmann, P. K., Yallop, B. D., Hohenkerk, C. Y.,: 1989, Astron. J., 97, 265.

Soma, M., Aoki, S.,: 1989, "Transformation from FK4 System to FK5 System", Paper submitted to Astron. Astrophys.

Standish, E. M. Jr.,: 1982, Astron. Astrophys., 115, 20.

Wielen, R., Lederle, T., Schwan, H.,: 1987, Corrections FK5 - FK4 to be Applied to the Published APFS for the Years 1984 to 1987, Astron. Recheninst., Heidelberg.

Yallop, B. D., Hohenkerk, C. Y., Smith, C. A., Kaplan, C. H., Hughes, J. A., Seidelmann, P. K.,: 1989, Astron. J., 97, 274. 